\title{
Tristimulus Weight Functions To Calculate Musts Color Coordinates From 10-nm Bandwidth Spectral Data
}

\author{
C. Montes a , J. Campos ${ }^{\text {b }}$, A. Pons ${ }^{\text {b }}$, F.J. Heredia ${ }^{\text {a }}$ \\ ${ }^{a}$ Área de Nutrición y Bromatología. F. Farmacia. Universidad de Sevilla. 41012 Sevilla. \\ ${ }^{\mathrm{b}}$ Instituto de Física Aplicada (CSIC). 28006 Madrid.
}

\begin{abstract}
Color measurement of red musts is affected by errors due to the instrument architecture. The influence of bandwidth in color coordinates errors is studied and a set of weighting functions for the color coordinates calculation, from $10 \mathrm{~nm}$ spectral data, is proposed.
\end{abstract}

Keywords: musts color, spectral bandwidth, weighting functions

\section{INTRODUCTION}

Must color measurement can be of great value during wine elaboration, both for evaluating grape maturation and for predicting color and chemical composition of wines. Tristimulus color results depend on measure conditions. Most of these measurements are done under different circumstances (industrial, country, laboratory environment) and, in many cases, large bandwidth instruments are used.

Although standard conditions have been defined for those parameters which influence the color measure result, such as observer, illuminant, illumination-observation geometry, bandpass and spectral measure interval, practical realizations differ from each other, so errors arise in calculating tristimulus values. These errors depend on the type of measure instrument and its characteristics of construction (Berns \& Petersen, 1988; Fairchild \& Reniff, 1991; Berns \& Reniff, 1997; Burns \& Berns, 1997). Furthermore, factors as temperature or relative humidity must be controlled to obtain single and repeatable measurements, because they may effect samples.

Color error as a function of measurement bandwidth has been studied for many sample types in several laboratories. In particular, color error in several types of food has been studied by our group, arising the conclusion that bandwidths larger than $5 \mathrm{~nm}$ produce a noticeable color error on many of them. Musts were one of those studied foods with a noticeable error in color coordinates for bandwidth larger than $5 \mathrm{~nm}$.

Within musts, color difference between measurements of the same object, with different instruments, are mainly due to the difference in measurement geometry and instrument bandwidth, since most instruments get spectral data rather than tristimulus color measurement properly. Differences in measurement geometry are not so important in this case because transmittance is the quantity used to determine color and the must is very transparent.

\section{EXPERIMENTAL PROCEDURE}

Twenty seven red must samples, corresponding to different stages along the grape maturation, and belonging to four harvest (1996-1999) have been studied. From them, five representative samples were selected to test the bandpass error elimination methodology. The rest of samples were used to obtain weighting functions.

A Hewlett-Packard HP8452 UV diode array spectrophotometer was used to scan the spectra of the samples. Tristimulus values were obtained from the visible transmittance values and chromatic coordinates were calculated for the CIE 1976 ( L*a*b*)-uniform color space, CIELAB (CIE, 1986). The method was applied over the whole visible spectrum, 380-780 nm, at $2 \mathrm{~nm}$ intervals, following the weighted-ordinate method. CIE standard illuminant A and CIE standard 
observer 1931 (field $<4^{\circ}$ ) were used in the color calculation. In order to study the bandwidth effect without influences of other experimental conditions. effective transmittance values were calculated by using the following equation:

$$
T_{u b l}=\frac{\int_{a b} T(\lambda) B(\lambda) d \lambda}{\int_{a b} B(\lambda) d \lambda}
$$

where $T(\lambda)$ is the sample's transmittance as measured by the spectrophotometer; $B(\lambda)$ is the slit function (triangular form is assumed) and $a b$ is the extension of the measure spectral interval. Transmittance values for a $10 \mathrm{~nm}$ bandpass have been calculated for all the samples and then color coordinates have been calculated using illuminant A and the CIE 1931 standard observer $\left(2^{\circ}\right)$ from 380 to $780 \mathrm{~nm}$ using a $10 \mathrm{~nm}$ summation interval.

There are mainly two approaches in the literature to avoid the bandwidth error in the calculation of color coordinates: Calculating weighting functions for the tristimulus functions or getting the $1-\mathrm{nm}$ radiance values from the experimental data. In the first case. ASTM (1995) recommends weighting factors $\mathrm{Wx}, \mathrm{Wy}, \mathrm{Wz}$, defined differently for 10 and $20 \mathrm{~nm}$ bandpass. These weighting factors already contain the product of illuminant $\mathrm{A}$ and the CIE 1931 standard observer $\left(2^{\circ}\right)$ for the respective interval and they have been prepared to correct spectral measurements errors due to bandpass effect. Color coordinates of our must samples have been calculated according to the ASTM procedure for a $10 \mathrm{~nm}$ bandwidth.

A method for correcting radiance data for bandpass error is proposed by Stearns and Stearns (1988). By applying this method to the samples, the calculated color coordinates should coincide with the 1-nm coordinates. The model estimates the zero bandwidth radiance data from the measured radiance, considering a $10 \mathrm{~nm}$ triangular and symmetrical bandpass and a measurement interval equal to the bandwidth. This method has been applied to our must samples to calculate color coordinates.

\section{RESULTS AND DISCUSSION}

Total color difference in $\left(\mathrm{L}^{*} \mathrm{a}^{*} \mathrm{~b}^{*}\right)$ as well as difference in lightness and chroma between 2-nm initial data and the coordinates calculated from the $10 \mathrm{~nm}$ bandpass values have been obtained, considering for the $10 \mathrm{~nm}$ bandwidth: uncorrected values. ASTM corrected values and values corrected by the Stearns \& Stearns method.

The ASTM weighting functions results are not satisfactory, because they produce an improvement of roughly 0.1 CIELAB unit in color difference with respect to the uncorrected value, and in many cases color differences in must samples are still higher than 0.5 CIELAB units after correction.

The Stearns \& Stearns method gives a better correction than the ASTM for most of the samples considered, producing a reduction of 0.2 or $0.3 \mathrm{CIELAB}$ units in color difference, but the correction is still insufficient and again many differences are larger than 0.5 CIELAB units.

To get a noticeable reduction in bandpass color error, different weighting functions were calculated according to the following criterion:

$$
\begin{aligned}
& x(\lambda, \Delta \lambda=1 \mathrm{~nm})=x(\lambda, \Delta \lambda \neq 1 \mathrm{~nm}) \times \mathrm{p}(\mathrm{x}) \\
& \mathrm{y}(\lambda, \Delta \lambda=1 \mathrm{~nm})=\mathrm{y}(\lambda, \Delta \lambda \neq 1 \mathrm{~nm}) \times \mathrm{p}(\mathrm{y}) \\
& \mathrm{z}(\lambda . \Delta \lambda=1 \mathrm{~nm})=\mathrm{z}(\lambda, \Delta \lambda \neq 1 \mathrm{~nm}) \times \mathrm{p}(\mathrm{z})
\end{aligned}
$$

The weighting functions are then defined as sets of values, one for every tristimulus function. The simplest expressions to calculate individual values for each are: 


$$
\begin{gathered}
p(x)=\frac{\sum_{\Delta \lambda} x(\lambda) * \mathrm{~T}(\lambda) * \mathrm{E}(\lambda) * \Delta \lambda}{10 * x(\lambda) * \mathrm{E}(\lambda) * \mathrm{~T}_{\Delta}(\lambda) * \Delta \lambda_{10}} \\
p(y)=\frac{\sum_{\Delta \lambda} y(\lambda) * \mathrm{~T}(\lambda) * \mathrm{E}(\lambda) * \Delta \lambda}{10 * y(\lambda) * \mathrm{E}(\lambda) * \mathrm{~T}_{\Delta}(\lambda) * \Delta \lambda_{10}} \\
p(z)=\frac{\sum_{\Delta \lambda} z(\lambda) * \mathrm{~T}(\lambda) * \mathrm{E}(\lambda) * \Delta \lambda}{10 * z(\lambda) * \mathrm{E}(\lambda) * \mathrm{~T}_{\Delta}(\lambda) * \Delta \lambda_{10}}
\end{gathered}
$$

where $\Delta \lambda$ is the spectral interval in which the slit function is defined; $x(\lambda), y(\lambda), z(\lambda)$ are the tristimulus functions, $E(\lambda)$ is the illuminant and $T(\lambda)$ is the sample transmittance.

These three equations were applied to the 1-nm and 10-nm spectral data of must samples, from the four harvests. For each harvest, arithmetic media was calculated, and, since no large difference was found from harvest to harvest, the arithmetic media of the four values was calculated again. The standard deviation of these averaged values was in the order of 2 parts per thousand. So, three suitable weighting functions were obtained to apply to general must samples (Fig. 1).

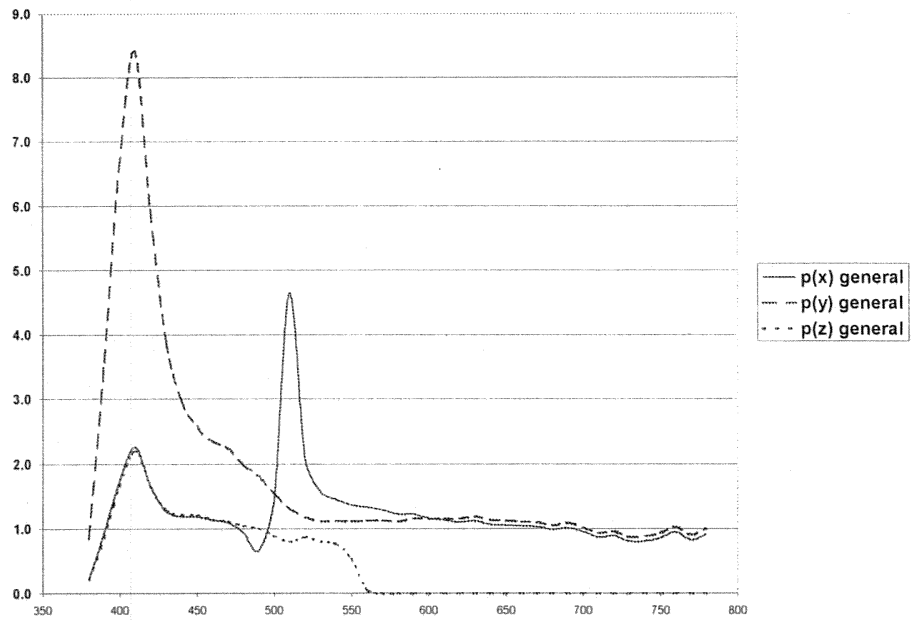

Fig. 1. Weighting Functions

The three weighting functions, $p(x), p(y), p(z)$ were analyzed, and each of them had a spectral zone where the correcting action is higher.

The function $p(x)$ has got a maximum value in 500-600 nm (red-orange zone). This zone coincide with the $x(\lambda)$ peak. The function has got another high value in the blue zone $(390-450 \mathrm{~nm})$ to correct the coordinate $\mathrm{b}^{*}$.

On the other hand, function $\mathrm{p}(\mathrm{y})$ is noticeable only in the blue zone $(380-480 \mathrm{~nm})$. The weighting function $\mathrm{p}(\mathrm{z}) \mathrm{had}$ a maximum for correcting blue wavelengths in the same zone than $\mathrm{p}(\mathrm{x})$. 
The three functions had a plane spectral zone $(580-780 \mathrm{~nm})$ where no corrections were necessary.

Color coordinates obtained by applying these weighting functions to a set of 5 samples of $10 \mathrm{~nm}$ transmittance values are much closer to the $2 \mathrm{~nm}$ values than those obtained by the other approximations (ASTM, Stearns). These samples, as mentioned before, were not used to derive the previous weighting functions

\section{CONCLUSIONS}

When bandpass is different from $1 \mathrm{~nm}$, errors arise in calculating color coordinates, although those errors are not noticeable when the bandwidth is lower than $5 \mathrm{~nm}$. There are two methods in the literature to try to correct those errors. Both have been tested in this work for must samples. However, by using ASTM weighting functions or applying the Stearns \& Stearns method there was no improvement in the higher bandpass values with respect to 1-nm bandpass.

To eliminate bandpass effect in tristimulus values it is necessary to calculate suitable weighting functions. A criterion for calculating these functions has been proposed. By applying these weighting function to must samples, it is possible to compare color coordinates obtained from 1 -nm spectral data to those obtained from 10-nm experimental values.

\section{REFERENCES}

ASTM. Annual book of ASTM standards, vol 06.01, 1995.

R.S. Berns, L. Reniff, "An abridged technique to diagnose spectrophotometric errors", Color Res. Appl. 22, pp 51-60, 1997.

R.S. Berns, K.H. Petersen. “Empirical modeling of systematic spectrophotometric errors," Color Res. Appl. 13, pp 243256, 1988.

P.D. Burns, R.S. Berns, "Error propagation analysis in color measurements and imaging", Color Res. Appl. 22, pp 280-289, 1997.

CIE. Colorimetry; Pub. CIE 15.2 Viena, Austria, 1986.

M.D. Fairchild, L. Reniff "Propagation of random errors in spectrophotometric colorimetry", Color Res. Appl. 16, pp 360$367,1991$.

E.I. Stearns, "Influence of spectrophotometer slits on tristimulus calculations", Color Res. Appl. 6, pp 79-84, 1981.

E.I. Stearns, R.E. Stearns, “An example of a method for correcting radiance data for bandpass error", Color Res. Appl. 13, pp 257-259, 1988.

W.H. Venable, "Accurate tristimulus values from spectral data", Color Res. Appl. 14, pp 261-267, 1989. 